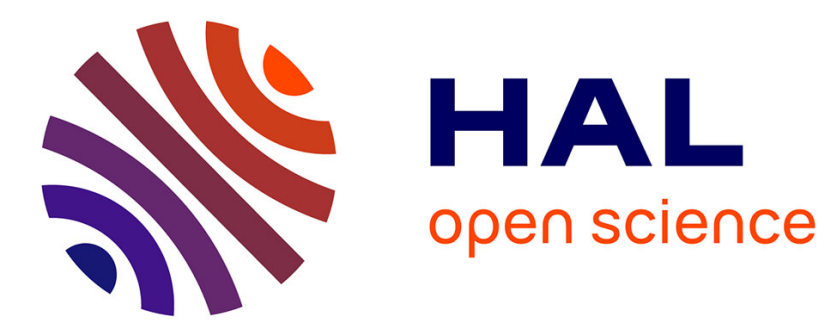

\title{
Dynamic light scattering study of the radical copolymerization of styrene-meta divinylbenzene
}

\author{
J.P. Munch, M. Ankrim, G. Hild, S. Candau
}

\section{To cite this version:}

J.P. Munch, M. Ankrim, G. Hild, S. Candau. Dynamic light scattering study of the radical copolymerization of styrene-meta divinylbenzene. Journal de Physique Lettres, 1983, 44 (2), pp.73-78. 10.1051/jphyslet:0198300440207300 . jpa-00232145

HAL Id: jpa-00232145

https://hal.science/jpa-00232145

Submitted on 1 Jan 1983

HAL is a multi-disciplinary open access archive for the deposit and dissemination of scientific research documents, whether they are published or not. The documents may come from teaching and research institutions in France or abroad, or from public or private research centers.
L'archive ouverte pluridisciplinaire HAL, est destinée au dépôt et à la diffusion de documents scientifiques de niveau recherche, publiés ou non, émanant des établissements d'enseignement et de recherche français ou étrangers, des laboratoires publics ou privés. 
Classification

Physics Abstracts

$51.20-61.40-82.35-82.90$

\title{
Dynamic light scattering study of the radical copolymerization of styrene-meta divinylbenzene
}

\author{
J. P. Munch, M. Ankrim, G. Hild (*) and S. Candau \\ Laboratoire d'Acoustique Moléculaire, Université Louis-Pasteur, \\ 4, rue Blaise Pascal, 67070 Strasbourg Cedex, France \\ (*) Centre de Recherches sur les Macromolécules, C.N.R.S., \\ 6, rue Boussingault, 67083 Strasbourg Cedex, France
}

(Reçu le 2 novembre 1982, accepté le 30 novembre 1982)

\begin{abstract}
Résumé. - Nous présentons les résultats d'une étude par diffusion laser d'une copolymérisation radicalaire (styrène divinylbenzène-AIBN-benzène). Le processus conduit à une gélification irréversible. La fonction d'autocorrélation de l'intensité lumineuse diffusée par le système à l'état de sol présente deux modes de décroissance indépendants. Le temps caractéristique de la relaxation lente suit la même variation temporelle que la viscosité macroscopique du système.
\end{abstract}

\begin{abstract}
We report a dynamic scattering study of a system undergoing irreversible gelation (styrene-divinylbenzene-AIBN-benzene). The autocorrelation function of intensity scattered from the sol exhibits two independently decaying modes. The slow-relaxation time follows the same time dependence as the macroscopic viscosity of the medium.
\end{abstract}

1. Introduction. - It is generally admitted that dynamic light scattering studies on longitudinal modes near the sol-gel transition do not provide important information on the gelation mechanism for the following reason : the collective diffusion coefficient, which characterizes the decay of the concentration fluctuations, is not very sensitive to the connectivity fluctuations occurring near the transition point [1]. In an irreversible gelation experiment, performed by radical copolymerization, the sol consists of branched polymers widely distributed in size and scattering power so that changes in the relative concentrations of different species are likely to contribute effects additional to those produced by the fluctuations in the total number density. This phenomenon has been theoretically investigated by Pusey, Fijnaut and Vrij (P.F.V.) for concentrated liquid suspensions of polydisperse hard spheres [2]. For such systems P.F.V. predicted that the light scattering correlation function consists of the sum of two independently decaying modes. The first mode is associated with the usual cooperative diffusion, while the second describes the exchange between species through self-diffusion. The existence of these two modes has been experimentally observed in microemulsions $[3,4]$, latex particles dispersions $[5,6]$ and silica spheres [7]. 
When a gelation is performed in the presence of a good solvent, the polymeric clusters formed near the gel point behave like hard spheres because of the excluded volume interactions, so that the gelling medium presents some similarities with the preceding systems. More specifically, we would expect a correlation function of scattered light to be composed of two independent modes with well separated decay times.

In this paper we report a dynamic light scattering study of the radical copolymerization process of styrene and m-divinylbenzene at $60^{\circ} \mathrm{C}$ in benzenic solution. The AZO-2,2' isobutyronitrile $(\mathrm{AIBN})$ was used as initiator [8].

2. Experimental. - Two experiments were performed on samples with the following compositions (in $\mathrm{g}$ per $100 \mathrm{~cm}^{3}$ of solution).

System A styrene : 18.44, meta DVB 1.07, AIBN 1.92

System B styrene : 19.91, meta DVB 0.061, AIBN 2.09.

The reactive elements were mixed in a glass tube, sealed after degassing under vacuum and then placed in a thermostat at $60^{\circ} \pm 0.1{ }^{\circ} \mathrm{C}$, on the stage of the photon correlation spectrometer.

The polymer volume fraction, at the gel point, was not measured but from the results of previous studies, one can infer that it was about 0.1 .

A Spectra-Physics argon-ion laser $(\lambda=488 \mathrm{~nm})$ was used in conjunction with a 48 channel clipped digital autocorrelator (Precision Devices and Systems LTD Malvern) for measuring the autocorrelation function of the light scattered at an angle of $90^{\circ}$. We used the so-called " heterodyne " detection in which a reference beam is superimposed onto the scattered beam. This allows us to analyse unambiguously the autocorrelation function of the photocurrent which is then proportional to the autocorrelation function of scattered intensity [9]. This is an important point in a gelation study, since a swollen polymeric network scatters light from both longitudinal concentration fluctuations and quasi-static swelling inhomogeneities [10], so that the homodyne spectrum contains a mixed mode associated with the beating of these two components.

The time required for the recording of one correlation function was of the order of $30 \mathrm{~s}$. The gelations considered here were rather slow $(\sim 15 \mathrm{~h}-25 \mathrm{~h})$ and we have not investigated the very close vicinity of the gel point. Therefore the change of properties of the system during a recording time was negligible as ascertained from the comparison between repeated successive recordings.

3. Results and discussion. - The shape of the autocorrelation function was found to be strongly dependent on the stage of the reaction. The very early stage is difficult to investigate because of the time required to attain thermal stability and also because the average decay time of the correlation function is very short, owing to the small size of the diffusing scatterers.

We started our observations about 1 hour after the beginning of the copolymerization. From that time, up to the gel point (to be discussed later), the shape of the autocorrelation function is strongly non exponential and is characterized by two widely separated decays.

This is demonstrated in figure 1 which shows a typical autocorrelation function at two time scales. Figure $1 a$ reveals the fast decay as displayed by the correlator with a sample time of $4 \mu \mathrm{s}$. The asymptotic behaviour of the correlation function is a straight line of non zero slope, which indicates the presence of a slow mode. The latter can be revealed by using a long sampling time $(\Delta \tau=300 \mu \mathrm{s})$ as shown in figure $1 b$. The corresponding decay time is obtained from a single exponential fitting procedure in which the content of the first channel is deleted as it contains most of the signal associated with the fast mode.

The determination of the fast decay times was obtained by fitting the correlation function to the sum of two exponentials using a least-squares program. As a matter of fact, the separation of the two decays was generally large enough so that the following form of the autocorrelation function could be used to fit the data when the correlator sample time was chosen to span the fast decay :

$$
C_{\mathrm{i}}(\tau)=A l^{-\tau / \tau_{\mathrm{F}}}+B\left(1-\tau / \tau_{\mathrm{s}}\right)+C
$$




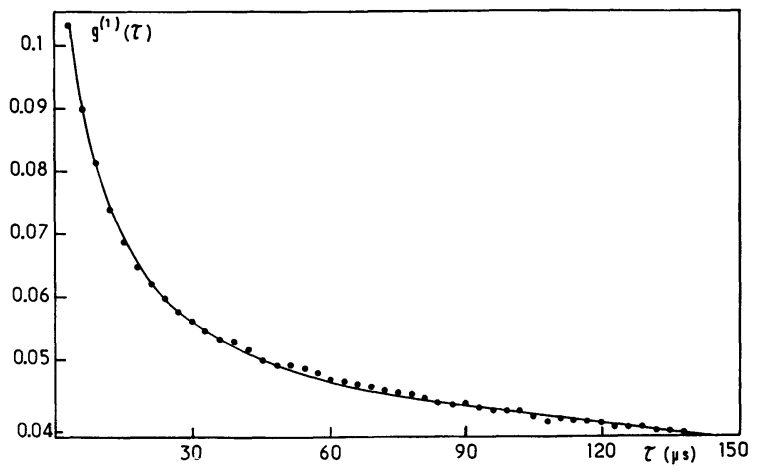

a)

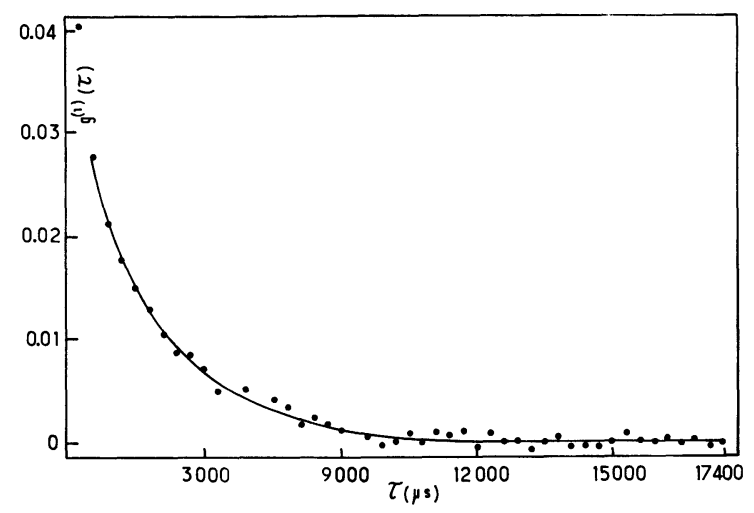

b)

Fig. 1. - Normalized autocorrelation functions for system $\left.\mathrm{A} ; t_{\mathrm{c}} / t=0.7 . a\right) \Delta \tau=4 \mu s$. The curve is a double exponential fit to the data. b) $\Delta \tau=300 \mu \mathrm{s}$. The curve is a single exponential fit to the data.

where $A, B$ and $C$ are constants and $\tau_{\mathrm{F}}$ and $\tau_{\mathrm{s}}$ are the fast and slow decay times respectively. The normalized autocorrelation function is :

$$
g^{(1)}(\tau)=\left(C_{\mathrm{i}}(\tau)-C\right) /(A+B)
$$

The time dependences of $\tau_{\mathrm{s}}$ and $\tau_{\mathrm{F}}$ obtained for system $\mathrm{A}$ have been plotted on figure 2 . The gel point $t_{\mathrm{c}}$ has been taken to be the time at which $\tau_{\mathrm{s}}$ becomes too long to be measurable. As a matter of fact, $t_{\mathrm{c}}$ can be rather well detected from the observations of the speckle pattern visible at very low scattering angles on a screen placed at a remote distance from the scattering cell. This pattern fluctuates randomly when the sample is in the sol state. A net slowing down is observed when approaching $t_{\mathrm{c}}$ beyond which the pattern becomes quasi-stationary. This also corresponds roughly to the stage where the sample does not flow when the cell is tilted. The observation of stationary speckle after $t_{\mathrm{c}}$ confirms the existence of microscopic inhomogeneities inside the gel. The experimental values of $t_{\mathrm{c}}$ were $15 \mathrm{~h} 10^{\prime}$ and $25 \mathrm{~h} 15^{\prime}$ for systems A and B respectively. Beyond $t_{\mathrm{c}}$ the autocorrelation function consists mainly of a continuous distribution of exponential decays plus a constant background corresponding to a quasi-static scattering. An analysis based on the method of cumulants [9] with the amplitude of the background taken as an adjustable parameter leads to a variance of the order of 0.2 . This variance decreases with time to reach eventually a value close to zero when the reaction is completed. 


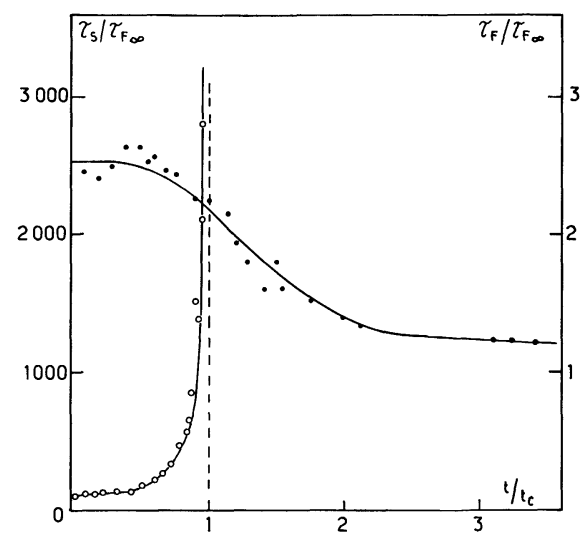

Fig. 2. - Variation of $\tau_{\mathrm{s}} / \tau_{\mathrm{F} \infty}$ and $\tau_{\mathrm{F}} / \tau_{\mathrm{F} \infty}$ versus $t / t_{\mathrm{c}}$ for system $\mathrm{A} ; \tau_{\mathrm{F} \infty}$ represents the asymptotic value obtained for $t / t_{\mathrm{c}} \rightarrow \infty ; t_{\mathrm{c}}=15 \mathrm{~h} 15^{\prime}$.

Inspection of figure 2 reveals some characteristic features which can be summarized as follows :

a) Fast mode. - Obviously, the fast decay can be attributed to the collective fluctuations characterized by a relaxation time :

$$
\tau_{\mathrm{F}}=\left(D_{\mathrm{c}} K^{2}\right)^{-1}
$$

where $D_{\mathrm{c}}$ is the cooperative diffusion constant and the magnitude of the scattering wavevector $K$ is given by :

$$
K=\frac{4 \pi n}{\lambda} \sin \theta / 2
$$

where $\theta$ is the scattering angle, $\lambda$ is the wavelength of the incident light in a vacuum and $n$ is the refractive index of the scattering medium.

As expected, $\tau_{\mathrm{F}}$ does not exhibit any abrupt transition at $t_{\mathrm{c}}$. It has already been demonstrated that the cooperative diffusion constant was nearly the same in a crosslinked gel and a semidilute polymer solution at the same concentration [10]. Near the gel point, the sol is formed of closely packed or even slightly interpenetrated branched aggregates, and the interconnection between these aggregates should not greatly affect the collective fluctuations. Nearly constant in the sol phase, $\tau_{\mathrm{F}}$ starts to decrease slowly around $t_{\mathrm{c}}$ to reach an asymptotic value $\tau_{\mathrm{F} \infty}$ after a long period $\left(t / t_{\mathrm{c}}>3\right)$. Such behaviour clearly indicates that the reaction still proceeds beyond the gel point, leading to a progressive decrease in the number of dangling chains attached by one end to the network. Also the insaturations (pendant double bonds) are slowly consummed in the later stage of the reaction [11]. As a consequence $D_{c}$ increases as shown previously in a study performed on swollen model networks containing controlled amounts of pendant chains [12]. Once the asymptotic value of $\tau_{\mathrm{F}}$ is reached, a further swelling of the gel up to its equilibrium value only produces a very small increase of $D_{\mathrm{c}}$.

$D_{\mathrm{c}}=15.5 \times 10^{-7} \mathrm{~cm}^{2} \mathrm{~s}^{-1}$ for a polymer concentration $c=0.195 \mathrm{~g} \mathrm{~cm}^{-3}$

$D_{\mathrm{c}}=16.7 \times 10^{-7} \mathrm{~cm}^{2} \mathrm{~s}^{-1}$ for $c=0.115 \mathrm{~g} \mathrm{~cm}^{-3}$ (at swelling equilibrium).

This result confirms the conclusions of a previous study performed on series of gels undergoing an osmotic deswelling and which also demonstrated a very weak sensitivity of $D_{c}$ to a change of concentration [13]. 
b) Slow mode. - From the considerations developed at the beginning of this paper, it seems reasonable to attribute the origin of this mode to the change in the relative concentrations of branched aggregates of different sizes. The PFV model predicts :

$$
\tau_{\mathrm{s}}=\left(D_{-} K^{2}\right)^{-1}
$$

where $D_{-}$is an undefined average value which PFV approximate for a fairly narrow particle size distribution to the self diffusion $D_{\mathrm{s}}$ of particles with radius equal to the mean of the distribution. Several authors $[3,4]$ have found that $D_{\mathrm{s}}$ in microemulsions varies like the viscosity. Despite the fact that the samples investigated here exhibit a rather large polydispersity mainly near the gel point [11], it is likely that the behaviour of the slow decay time is still correlated to the viscosity of the medium. In previous studies [14-15] the time dependence of the viscosity was found to obey a power law of the form :

$$
\eta \sim\left(\frac{t_{\mathrm{c}}-t}{t_{\mathrm{c}}}\right)^{-s}
$$

where $s$ is of the order of 1 . In the close vicinity of $t_{\mathrm{c}}$, that is at $\frac{t_{\mathrm{c}}-t}{t_{\mathrm{c}}} \sim 2 \times 10^{-2}$ a cross-over is observed beyond which $\eta$ follows another power law with $s \sim 0.78$ [15]. Figure 3 shows a log$\log$ plot of $\tau_{\mathrm{s}}^{-1}$ versus $\left(t-t_{\mathrm{c}}\right) / t_{\mathrm{c}}$ for the system $\mathrm{A}$. The data fit a straight line with a slope equal to -1.1 , the coefficients of correlation being of the order of 0.988 . The same exponent is found for system B. As mentioned before, there is some uncertainty on the value of $t_{\mathrm{c}}$ so that the behaviour of $\tau_{\mathrm{s}}$ close to the gel point cannot be characterized. However, this uncertainty does not strongly affect the exponent of the power law $\tau_{\mathrm{s}}^{-1}=f\left(\frac{t-t_{\mathrm{c}}}{t_{\mathrm{c}}}\right)$. For instance, shifting $\left(t-t_{\mathrm{c}}\right) / t_{\mathrm{c}}$ from + and -0.02 leads to exponents respectively equal to 1.05 and 1.2 with a coefficient of correlation 0.986 .

This paper was designed to provide a description of the time dependences of the characteristic decay times of the autocorrelation function of light scattered from a system undergoing irreversible gelation. In a subsequent paper we will present a more detailed analysis which will include the behaviour of the amplitudes of the two modes and their dependences on the concentration of the styrene and of the DVB.

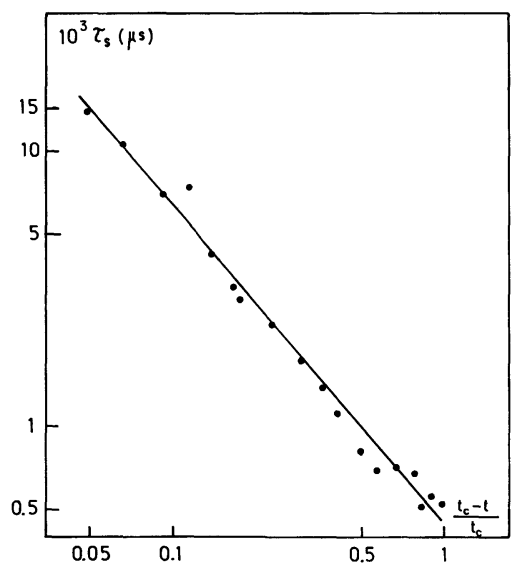

Fig. 3. - Log-log plot of $\tau_{\mathrm{s}}$ versus reduced time $\frac{t-t_{\mathrm{c}}}{t_{\mathrm{c}}}$ for system A; $t_{\mathrm{c}}=15 \mathrm{~h} 15^{\prime}$. The straight line is the best linear fit to the data. 


\section{References}

[1] De Gennes, P. G., Scaling Concepts in Polymer Physics (Cornell University, Ithaca) 1980.

[2] Pusey, P. N., Fijnaut, H. M., VRIJ, A., J. Chem. Phys., To be published.

[3] Cebula, D. J., Ottewill, R. H., Ralston, J., Pusey, P. N., J. Chem. Soc. Faraday Trans. 77 (1981) 2585.

[4] Cazabat, A. M., Chatenay, D., Langevin, D., Meunier, J., Léger, L., Proceedings of the International Symposium in Surfactant in Solution, Lund (Sweden), June 1982.

[5] Bauer, D. R., in Polymer Colloids, edited by Fitch R. M. (Plenum, New York) 1980.

[6] Bauer, D. R., J. Phys. Chem. 84 (1980) 1592.

[7] Kops-Werkhoven, M. M., Mos, H. J., Pusey, P. N. and Fijnaut, H. M., Chem. Phys. Lett. 81 (1981) 365.

[8] Okasha, R., Hild, G. and Rempp, P., Eur. Polym. J. 15 (1979) 975.

[9] See for instance : Photon Correlation and Light Beating Spectroscopy, Ed. Cummins, H. Z. and Pike. E. R. (Plenum, New York) 1974.

[10] Munch, J. P., Candau, S., Herz, J. and Hild, G., J. Physique 38 (1977) 971.

[11] Hild, G. and Rempp, P., Pure Appl. Chem. 53 (1981) 1541.

[12] Bastide, J., Picot, C. and Candau, S., J. Polym. Sci. Polym. Phys. Ed. 17 (1979) 1447.

[13] Bastide, J., Duplessix, R., Picot, C. and Candau, S., Submitted to Macromolecules.

[14] Gordon, M. and Roberts, K., Polymer 20 (1979) 681.

[15] Adam, M., Delsanti, M., Okasha, R. and Hild, G., J. Physique-Lett. 40 (1979) L-539. 